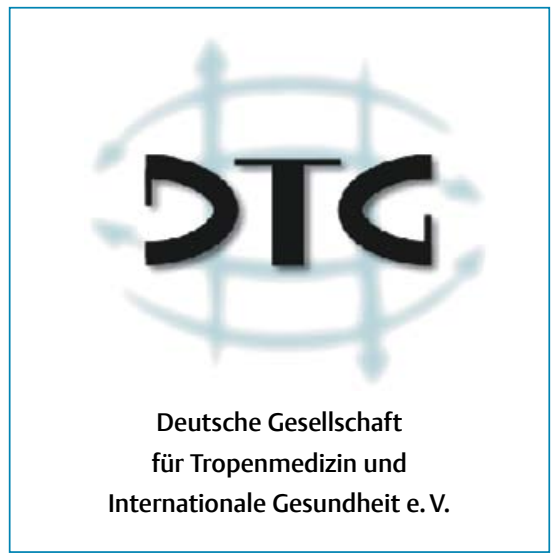

wir wünschen Ihnen ein wenig spät, aber dennoch sehr herzlich ein gutes Jahr 2013 und hoffen, dass Sie unserer Fachgesellschaft auch in diesem Jahr treu bleiben.

Im vergangenen Jahr hat der neu konzipierte Ausschuss Global Health unter dem Vorsitz von Albrecht Jahn aus Heidelberg bereits 2 -mal getagt und sich mit seinem eigenen Profil und seiner Struktur befasst. Fünf Schwerpunkte haben sich die Mitglieder gesetzt:

- Umwelt und Gesundheit

- Disease Control und Gesundheitssystementwicklung

- Gesundheit und Menschrechte

- Global Health Policy

- Internationale Partnerschaften und Netzwerke

Wichtiges Thema dieses Ausschusses ist auch das nächste Rahmenprogramm der EU für 2014-2020 („Horizon 2020“), das mit einem Budget von $80 \mathrm{Mrd}$. Euro alle Forschungs- und Innovationsprogramme der EU zusammenfasst. Für gemeinsame Interessen der Europäer im Bereich Klimawandel, Food Safety, erneuerbare Energien und den Herausforderungen einer alternden Gesellschaft stehen immerhin $31 \mathrm{Mrd}$. Euro zur Verfügung. Unter http://ec.europa. eu/research/horizon2020/index_en.cfm finden Sie viele Informationen zu diesem Programm, mit dem sich der DTGAusschuss auch kritisch auseinandersetzen wird.

Auch auf dem VIII. Europäischen Kongress für Tropenmedizin vom 10.-13.09.2013 in Kopenhagen wird die DTG wieder ein Symposium zum Thema „North-South Research" mitgestalten - die in der DTG vertretenen Institute werden in 5-6 Beiträgen ihre Forschungskooperationen mit den Partnern in den Tropen darstellen, möglichst aus der Perspektive der Partner vor Ort. Homepages dazu: www. ectmih2013.dk und www.festmih.eu.

\section{Aus der Vorstandssitzung}

Unsere AWMF-Leitlinie Schistosomiasis steht zur Aktualisierung an und gemeinsam mit den Gastroenterologen werden wir eine Leitlinie zur Reisediarrhö entwi- ckeln. Schon auf der Klausurtagung im vergangenen Sommer wurde die Entwicklung einer Leitlinie zur Untersuchung nach Tropenaufenthalten diskutiert, da der G35 doch nur sehr rudimentäre Vorgaben macht. Auf der Vorstandssitzung haben wir dazu jetzt einen Beschluss gefasst.

Um eine Erhöhung der Mitgliedsbeiträge auf 50 Euro werden wir angesichts unserer Vorhaben und auch in Folge der Erweiterung der Homepage und des Serviceangebots unserer Gesellschaft in diesem Jahr nicht herumkommen - festgesetzt wurden 50 Euro jährlich, ein entsprechender Vorratsbeschluss wurde ja bereits in Heidelberg auf der Mitgliederversammlung gefasst.

In die überarbeitete Homepage sollen ein moderierter Chatroom für Mitglieder, eigene Seiten für Nachwuchs und Ausschuss Global Health sowie ein Verzeichnis für Famulatur, PJ und andere Tätigkeiten eingebaut werden. Der Vorstand bestätigte außerdem einstimmig Jakob Cramer aus Hamburg als neuer Vertreter der DTG im Editorial Board der FTR. Er ist Nachfolger von Gerd Burchard.

Die DTG ist auch Mitglied in der neu gegründeten Arbeitsgemeinschaft wissenschaftlicher Fachgesellschaften in der Infektionsmedizin (AFIM; sonstige Mitglieder: DAIG, DGHM, DGI, DGPI, GIV, PEG). Deren Seiten, www.a-fim.de, sind bereits im Aufbau. Dort können zukünftig aktuelle Mitteilungen, Stellungnahmen, Leitlinien und Expertenlisten veröffentlicht werden.

Die nächste Vorstandssitzung wird im April stattfinden.

Auch wenn es noch einige Zeit hin ist, so möchten wir Sie jetzt schon bitten, sich den Termin der nächsten DTG-Tagung am 13.-14.03.2014 in Düsseldorf vorzumerken.

\section{God's Surgeons in Africa}

Ich bekam eine E-Mail von einem, Schüler', den ich während meiner Tätigkeit in Nigeria als jungen Arzt kennenlernte und in Innerer Medizin und vor allem auch im Ultraschall ausbilden konnte. Er ist heute in Mbingo, Kamerun, im Baptist Hospital als Chirurg tätig und 
aktiver Tutor im dort laufenden Ausbildungsprogramm für junge Chirurgen: Deren Qualifikation befähigen sie zwar, zukünftig alle wichtigen Eingriffe kompetent durchzuführen - formal reicht sie jedoch nicht aus, um in einem anderen Land wie Saudi-Arabien, Südafrika oder Großbritannien als Chirurg zu arbeiten. Für diese Kollegen entfällt damit die Möglichkeit, des Geldes wegen ihre Heimat und den Ort ihrer Ausbildung zu verlassen. Träger ist die Pan African Academy of Christian Surgeons (PAACS), die 43 Residents in ganz Afrika ausbildet und deren bisher 28 Graduierte noch immer in ihren Heimatländern chirurgisch tätig sind. Das Jahresbudget der Organisation für 2011 betrug 550000 US-Dollar. Über meinen ehemaligen Assistenten wird in einem sehr anschaulichen Artikel über dieses und ähnliche Projekte Folgendes geschrieben:

Ngoe Nesoah, a surgical resident at Mbingo who went to medical school in Nigeria, told me that nearly all of his classmate who trained in surgery have since left the country, many for Saudi Arabia. „Corruption has invaded the core leadership of Africa,“ he said. „And medicine is not unique." Nesoah is tall and keeps a thick mustache. He's well-read, and wears glasses with clear plastic rims and soccer flats with his scrubs. He grew up in both Cameroon and Nigeria, and has an outstanding serenity about him. „My mother and father aren't happy that I'm here,“ he told me. „My mother thinks

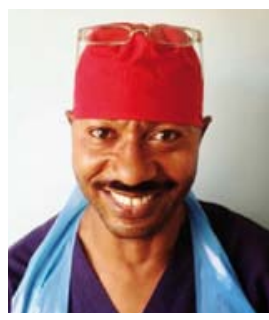

Der ganze Artikel und das sehr liebenswerte Bild des Kollegen Ngoe Nesoah auf: www.theatlantic.com/ health/archive/2012/ $12 /$ gods-surgeons-inafrica/266635/

I should be, as a doctor, I should be driving a Benz car and living in a big flat,“ Nesoah said, his arms crossed over his surgical gown. „But it's not her weakness. It's a reflection of the society. If you ask an African, ,why you should go to medical school?' He says, ,I want to be rich, I want to send my children to England to study.“

Wir grüßen Sie herzlich aus München und Hamburg
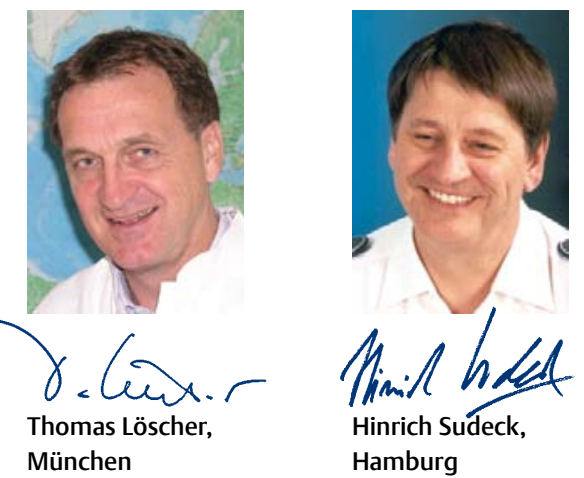

Fragen an die DTG

Warum gibt es die Empfehlung, eine Gelbfieberimpfung nicht während einer laufenden Desensibilisierungsbehandlung durchzuführen?

In der Literatur findet sich die Aussage, dass während einer subkutanen Desensibilisierungsbehandlung die Applikation anderer, möglicherweise allergisierender Substanzen unterbleiben sollte. Die AWMF-Leitlinie zur spezifischen Immuntherapie differenziert dies folgendermaßen: Zwischen einer SCITInjektion und einer planbaren Impfung sollte der Abstand mindestens eine Woche betragen. Impfungen sollten daher in der Erhaltungsphase der SCIT durchgeführt und zwischen 2 mit 4-wöchigem Abstand applizierten SCIT-Injek- tionen gegeben werden ( $D, 5)$. Sofort notwendige Impfungen (z.B. Tetanus nach Verletzungen) können jederzeit erfolgen.

Warum empfiehlt die DTG, anders als die entsprechenden Fachgesellschaften beispielsweise in England oder die WHO, für Indien keine Malariaprophylaxe?

Auf diese Frage einer Reisegruppe antwortet Professor Hans Dieter Nothdurft: Der Grund für diese Empfehlung, die auf einer seit Jahren bestehenden Expertenmeinung aus Deutschland, Österreich und der Schweiz beruht, liegt in der Analyse der aus Indien importierten Malariafälle, die vorwiegend Malaria-
tertiana-Fälle sind und demnach durch eine gängige Chemoprophylaxe nicht zu verhindern wären. Die Risiko-NutzenAnalyse einer Malariachemoprophylaxe für Indien zeigt, dass bei der geringen registrierten Fallzahl an Malaria-tropica-Patienten aus Indien mehr Schaden als Nutzen bei einer prophylaktischen Malariamedikamenteneinnahme zu erwarten wäre. Daher empfiehlt die DTG für Indien gute Mückenschutzmaßnahmen (z.B. Repellents) und bei einer mindestens einwöchigen Aufenthaltsdauer die vorsorgliche Mitnahme eines Notfallmedikaments (für wenigstens einige der Reiseteilnehmer); insbesondere bei Reisen abseits medizinischer Versorgung. 